\title{
Procedure End Day
}

National Cancer Institute

\section{Source}

National Cancer Institute. Procedure End Day. NCI Thesaurus. Code C117532.

The final day of the procedure. 